\title{
ON FORMATION OF STRUCTURES: DESIGN EXAMPLES AND DISCUSSION
}

\author{
Hou, Yuemin (1,2); \\ Ji, Linhong (2) \\ 1: Beijing information Science and Technology; \\ 2: Tsinghua University
}

\begin{abstract}
This paper discusses the formation of structures by taking the process of gene transcription and translation as the template. The hypothesis of this paper is that the gene transcription and translation process can describe the formation of structures both in engineering design and in biology. The paper first presents design examples including integrated circuit (IC) chambers, flapping wings of bird robots, and typical mechanisms and formulate the formation patterns of the design process as four steps: information interpretation, selection of building blocks, the connection of building blocks, and formation of structures. The key step of the formation process is to assemble building blocks for structures both in engineering and in biology. Building blocks in biology are amino acids while they are structures in design. The autonomous degree of the formation process depends on the level of building blocks. The reuse degree of the building blocks depends on the level of building blocks too. In biology, structures of proteins are self-organized, so one way towards design automation is to use lower-level building blocks.
\end{abstract}

Keywords: Formation of structures, Design process, Design methodology, Bio-inspired design / biomimetics

\section{Contact:}

Hou, Yuemin

Beijing Information Science and Technology University

Mechanical Engineering

China, People's Republic of

houyuemin@tsinghua.edu.cn

Cite this article: Hou, Y., Ji, L. (2021) 'On Formation of Structures: Design Examples and Discussion', in Proceedings of the International Conference on Engineering Design (ICED21), Gothenburg, Sweden, 16-20 August 2021. DOI:10.1017/ pds. 2021.490 


\section{INTRODUCTION}

How are structures formed? In biology, the growth process of structures is self-organized and undergoes two phrase transfers at the macro level: first from the information to materials and secondly from materials to structures, namely gene transcription and translation. The concept of gene transcription and translation describes the process from DNA information to protein structures, it is reasonable to use them to explain the formation of structures. This paper discusses the formation of structures by taking the process of gene transcription and translation as the template. The hypothesis of this paper is that the gene transcription and translation process can describe the formation of structures in engineering design. In the following sections, the paper discusses design examples including integrated circuit (IC) chambers, a bird robot with flapping wings and typical mechanisms and formulates the formation patterns as four steps. Related works are discussed in Section 3 and research findings are concluded in Section 4.

\section{DESIGN EXAMPLES AND DISCUSSION}

In this section, design examples are analysed by analogy with the process from DNA to proteins.

\subsection{Four steps from the information to structures}

In biology, the basic step from DNA to proteins is gene transcription and translation, by which a gene's DNA (Deoxyribonucleic acid) is read to produce mRNA (messenger ribonucleic acid) and then mRNA becomes translated by ribosomes to manufacture proteins (Kimmel and Buelke-Sam, 1994). mRNA has to be transported from the nucleus to the cytoplasm for protein synthesis. In the cytoplasm, mRNA along with transfer RNA (tRNA) and ribosomes work together to produce proteins, which is called translation. To put briefly, the process can be simply summarized as four steps.

- $\quad$ Step 1: interpret information: transcription of DNA.

- $\quad$ Step 2: define a sequence of building blocks: a sequence of amino acids is defined.

- Step 3: connect building blocks: produce the string of amino acids that makes up a protein.

- Step 4: formation: the string of amino acids are formed into a particular form for a particular functionality.

Step 1 to Step 2 is to transform the information into materials, while Step 3 is to form raw structures and Step 4 is to refine the structure into a particular form.

Similar steps are used to describe the process of formation of structures in design in this section. In this section, the design examples of IC chambers, the flapping wing of a bird robot, and several typical mechanisms are discussed. These design examples show how structures are formed. Three design strategies are used in the design of these products: pattern-oriented strategy, experiment-based decision, and kinematic-governed computing.

\subsection{Pattern-oriented strategy}

Pattern-oriented strategy is to take typical structural patterns as building blocks and then assembling these building blocks into a product. The process includes several main steps. The first step is to decompose main functions into a set of subfunctions that are relevant to known working principles. The second step is to select or define relevant working structures, i.e. structure patterns. The third step is to select or define the connection methods between the selected working structures, i.e. to decide connection patterns. The fourth step is to instantiate structures and connection patterns with structural parameters based on analysis and experiments and produce an engineering drawing. The fifth step is to test and validate the structural parameters. The last step, which usually not included in a design process, is to manufacture the product. Direct mapping between functions and structures is common in real engineering design because engineers usually design products based on existed function-structure mapping examples. Behaviour analysis is necessary for determining the detail dimensions but not necessary for a sketch of the structure.

Design examples of IC equipment illustrate this pattern-oriented design process. To produce a layer of film on a wafer for chips is the main function of the process chamber of integrated circuit (IC) equipment. This main function is decomposed into several sub-functions that are relative to two working principles: physical vapour deposition (PVD) and chemical vapour deposition (CVD). 
Structure patterns that are predefined on the computer are selected to produce two types of equipment automatically, as shown in Figure 1.

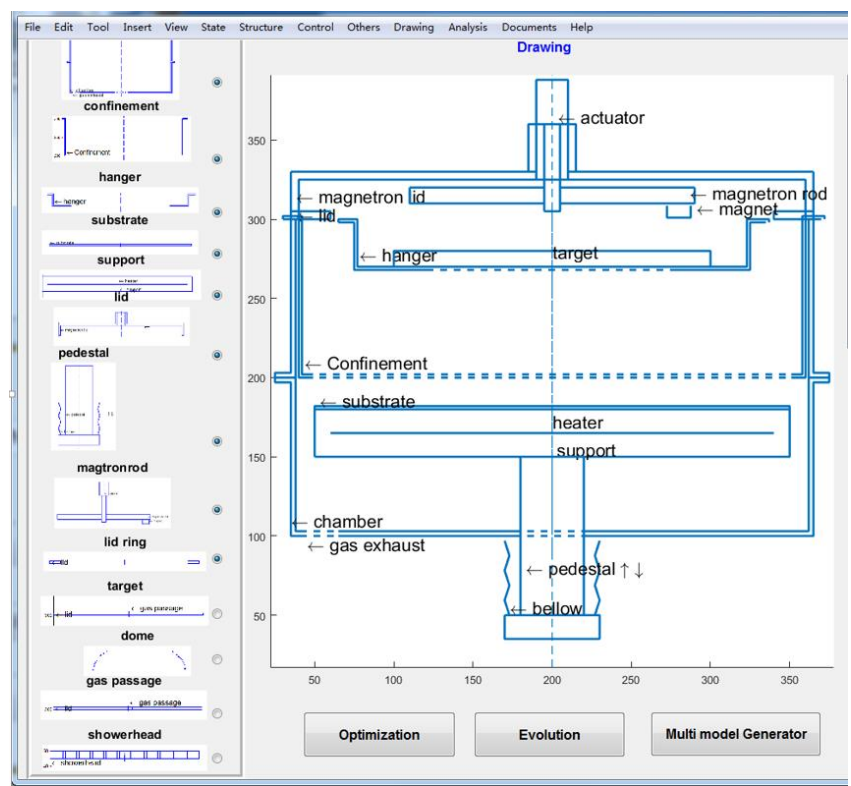

(a)

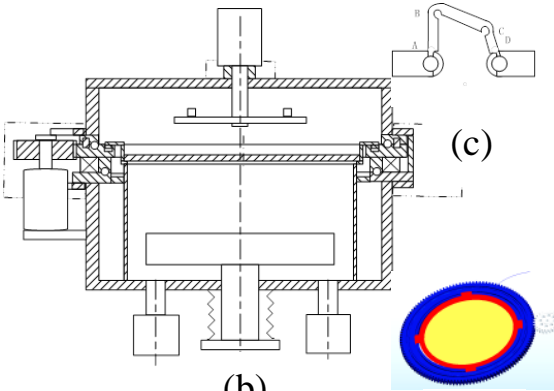

(b)

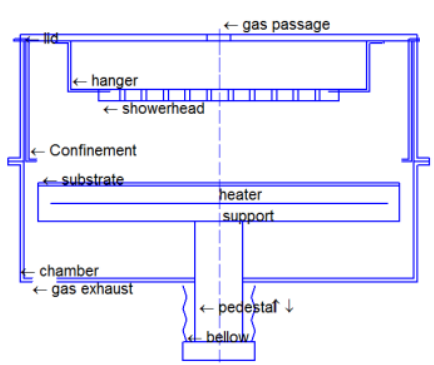

(e)

(d)

Figure 1. Structure patterns as working structures. (a) PVD chamber; (b) the embodiment of the PVD chamber; (c) the linkage mechanisms; (d) the rotary target; (e) CVD chamber

If to ask design engineers of IC equipment manufacturing company what is the design problem, they would answer that there is no design problem and the key problem is process simulation. The reason is that engineers use a pattern-oriented design strategy and there is no complete behaviour knowledge available for this kind of equipment. Analysis for detail design is necessary but there is no exact mapping between behaviours and structures. The detail parameters of a chamber are usually determined based on existing equipment. Engineers use simulation software to check the process parameters. For example, a cylindrical chamber is selected based on existing products and the diameter of the cylinder depends on the diameter of the wafers deposed, while the height of the chamber depends on the deposition rate and the film uniformness. The far the distance between the target material/showerhead and the substrate, better is the film uniformness, but the deposition efficiency of the film is lower. Engineers would trade off between these two criteria and then set the height of the chamber and the distance between the target material and the substrate. It would be perfect to establish an optimal model to find optimal structure parameters, but it is not the case in real engineering design. It is rather complex even impossible to establish mathematic functions to govern behaviour-structure mapping since there is no complete knowledge available so far for such a relationship. Figure 1 shows the draft drawing of the embodiment of a PVD chamber without setting structural parameters. Structural parameters are not strictly governed by equations but are determined based on handbooks as well as expertise, particularly on process simulation.

The basic arrangement of chambers in Figure 1 is produced by selecting structure patterns. Changes can be made for better uniformness of deposed films. The major change is to make the target rotate around its centre and to make the target centre not coincide with the axis of the magnetron for better deposition uniformness. This change leads to more complex structures for sealing, motion transmission, bearings, shaft, driver, bolt connections, and assembly of additional contact pairs. The assembly for the rotary target is shown in Figure 1(d). The second change to improve the uniformness of the deposited film is to use a flexible four-bar linkage mechanism that can achieve different profiles at different positions while it rotates around the magnetron rod frame so that the magnet field change leads to the electric field change. There are no existed examples for reference for both the design of a rotary target and a flexible linkage, so they are not produced by the computer system. Instead, they are designed by the designer. Figure 1(c) shows the flexible four-bar linkage.

This example shows how products are produced with a pattern-oriented design strategy. This strategy is also used as a key solution for the current KBE technology and design automation methods, in 
which structural templates are predefined as working structures. The most difficult part in the design process is the innovative design of the rotary part and the structure of this part cannot be predefined.

\subsection{Experiments-based decision}

In mechanical design, the syntheses of mechanisms are the key to meet kinematic requirements. Cam mechanisms, linkage mechanisms, and the like are basic working structures, while shaft connection, bearing connection, bolt connection, and key connection are basic connections. The key to the design is the syntheses of mechanisms including the profiles of mechanisms and dimensional parameters.

The second example presented here is the design of flapping wings of a bird robot. Linkage mechanisms are widely used for flapping wings. We started the design process with a selection of mechanisms based on existing examples. A multi-bar linkage that enabling flapping morphing wings is selected as a design reference. But changes are required to extend the angles between the up position of flapping motion and the down position of flapping motion to imitate real bird's motion. Two changes are made. First, the topology of the linkage mechanism is changed by using a slide pair to replace a revolute pair to enhance the strength of the connection pair. Secondly, the lengths of the bars are determined through experiments and simulation through six tests and simulations for searching proper parameters that made the linkage mechanism flap in a similar way with real birds. Figure 2 illustrates the topology of the flapping wing, the motion simulation, and the embodiment of the bird robot.

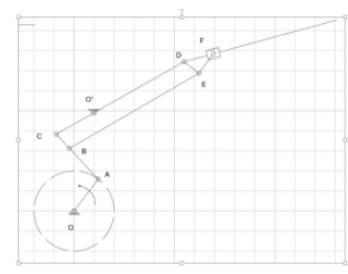

(a)

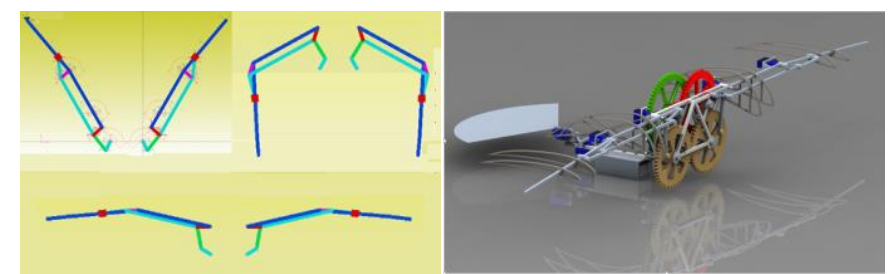

(b) (c)

Figure 2. The design and experiment of a bird robot with flapping wings. (a) The topology of the linkage mechanism; (b) motion simulation; (c) the embodiment

Theoretically, the parameters of bars can be calculated using a group of kinematic equations and mechanics equations. But in practice, the parameters are usually determined by estimation and experiments, as in the case presented here. The strength of the bars and connection pairs are calculated for detail design, but only dimensional parameters are required to meet the motion requirement in the synthesis phase. The key task of the design of the flapping wing is to set proper topology and determine proper lengths of bars. Calculation and optimization are theoretically good methods but they cost more time and energy on establishing optimization models and solving the equations. This is why engineers usually determine the design parameters through simulation/experiment rather than through optimization methods. Detail structural parameters are determined both by behaviour computing and estimation in engineering design practice. Key parameters need calculation based on equations while other parameters usually are decided based on estimation. This example shows that experiment-based decision in design is a reliable method and it is less complex than computing-based analysis.

\subsection{Kinematics-governed computing}

In the case of the desired set of positions, e.g. velocities and acceleration at definite points of time are stipulated, mechanisms are widely used and the geometry of members of mechanisms is mathematically determined by the specified motion. Conventionally, different equations are used for both the synthesis and kinematic analysis of different types of mechanisms.

For example, a set of positions at definite points of time are stipulated, then the profile of a cam is calculated based on a set of kinematic equations, which defines the relationship between specified behaviour and a cam profile. The base circle $r_{0}$ of a cam, which is the radius of the smallest circle from the cam centre through the cam profile curve, can be decided based on force constrains and manufacturing constraints. The force constraint is that the pressure angle $\alpha$, which is the angle at any point between the normal to the pitch curve and the instantaneous direction of the follower motion, must be less than or equals to a certain value, i.e. $\alpha \leq \alpha_{0}$. $\alpha_{0}$ is the limit. The manufacturing constraint is

$$
r_{0}>(1.6-2) \times r
$$


where $r$ is the radius of the camshaft. $\alpha$ can be calculated by the following equation.

$$
\left.\tan \alpha=\left[\left(\frac{d s}{d \delta}\right)-e\right] /\left(r_{0}^{2}-e^{2}\right)^{1 / 2}+s\right]
$$

where, $\delta$ is the rotation angles of the cam, $s$ is the displacement of the follower and $s$ is a function of the rotary angle of $\delta$, and $e$ is the offset of the follower from the rotary centre of the cam.

In this paper, linkage mechanisms and cam mechanisms are produced by repeatedly using four algorithms: Rotation, Centre, Connection and Numerical Differentiation.

The Rotation algorithm is used to generate the profile of a mechanism. By consequently rotating the discrete points $P_{\mathrm{i}}(x, y)$ with $\delta_{\mathrm{i}}$, cam profiles can be generated. Different kinds of cam profiles can be obtained by changing the working positions $x i$ and the rotational centre of the cams. Most important, this rule is also used to generate the profile of four-bar mechanisms. The mathematical representation of the Rotation algorithm is

$$
P(x, y)=\left[\begin{array}{ll}
x_{1} & y_{1} \\
x_{2} & y_{2} \\
\cdots & \cdots \\
x_{n} & y_{n}
\end{array}\right], T_{R}=\left[\begin{array}{cc}
\cos \left(\delta_{i}\right) & -\sin \left(\delta_{i}\right) \\
\sin \left(\delta_{i}\right) & \cos \left(\delta_{i}\right)
\end{array}\right], \text { and } P^{\prime}(x, y)=P(x, y) \cdot T_{R}
$$

where $P(x, y)$ is a set of specified position of the follower and $P^{\prime}(x, y)$ is the point of the cam profile while the cam rotates the angle $\delta_{\mathrm{i}}$.

The Centre algorithm is used to find the position of joints of linkage mechanisms as well as the joints of the followers of cam mechanisms. The relative displacement of two joints is obtained first through the Rotation operation and then the centre of the rotation can be obtained as the positions of joints.

In the case of producing cam profiles, the Centre rule determines the type of cam. A planar cam is produced when taking a point as the rotational centre of the cam, while a cylindrical cam is produced when taking a straight line as the rotational centre for the same $s(\delta)$ curve. The mathematic representation of Centre rule is

$$
x^{2}+y^{2}=r^{2}
$$

where $r$ is the radius of the rotation.

The Connection rule means linking points to form curves or lines and connecting masses to create forms. Equations can be used to produce parameter values but not the structure itself. The profiles of the mechanisms are produced by the Connection rule.

The kinematic analysis is conducted based on Numerical rule. In this case, both cam mechanisms and planar linkage mechanisms can use the same equations. The mathematic representation of Numerical Differentiation rule is

$$
y^{\prime}=\frac{y_{x+h}-y_{x-h}}{2 h}, y^{\prime \prime}=\frac{y_{x+h}+y_{x-h}-2 y_{x}}{h^{2}}
$$

where, $y^{\prime}$ and $y^{\prime \prime}$ are the first and second derivative, $x$ is the value at which the derivative is calculated, $h$ is a small number, $y_{\mathrm{x}+\mathrm{h}}$ is function evaluated at $x+h$, and $y_{\mathrm{x}-\mathrm{h}}$ is function evaluated at $x-h$.

Design examples presented here are designing mechanisms to achieve reciprocating motions. The input data is the working states of the followers or the bars, represented as a sequence of points. Other input data include the types of the mechanisms and basic parameters, such as initial base circle radius $r_{0}$ of a cam. The whole process is automatically conducted.

Example 1: Design of the cam profile of a translating follower cam mechanism. The rotation centre is a point. The Rotation rule and Connection rules are used. The result is shown in Figure 3a.

Example 2: Design of the cam profile of a swing follower cam mechanism. The Rotation rule and Connection rule are used. The rotation centre is a point. The result is shown in Figure $3 \mathrm{~b}$. The difference between the example 1 and 2 is the input points. They are a straight line in Example 1, while they are an arc in Example 2. 
Example 3: Design of the cam profile of a cylindrical cam mechanism. The Rotation rule and Connection rule are used. The result is shown in Figure 3c. The difference between example 1 and example 3 is the rotational centre of the cam. The centre is a point in Example 1, while it is a straight line in Example 3.

Example 4: Design of the cam profile of a crank-slide mechanism. The Rotation, Centre, and Connection rules are used. The result is shown in Figure 3d. The difference between example 1 and 4 is the rotational centre of the points. It is a fixed centre in Example 1, while it is in motion in Example 4.

Example 5: Design of the profile of a crank rocker mechanism. The Rotation, Centre, and Connection rules are used. The result is shown in Figure 3e. The difference between the example 4 and 5 is the rotational centre. It is on the crank in Example 4, while it is on the link in Example 5.

The size of a cam profile grows until the pressure angle is less than or equals to $[\alpha 0]$, as shown in Figure 4(a)-4(c). When the points are replaced by points or tine volume of masses, the cam can be created with hybrid materials, as shown in Figure 4(e) to Figure 4(g).

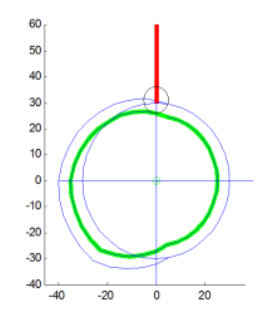

(a)

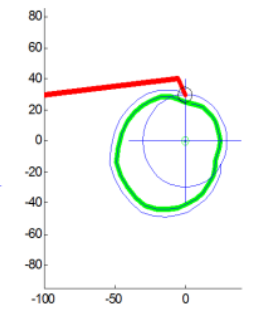

(b)

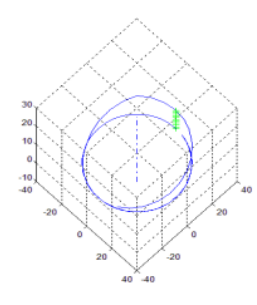

(c)

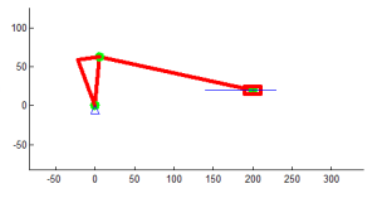

(d)

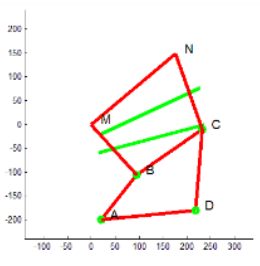

(e)

Figure 3. Mechanism profiles generated by four simple rules. a. planar cam with translating follower; b. planar cam with swing follower; c. cylindrical cam; $d$. crank slide mechanism; e. crank rocker mechanism

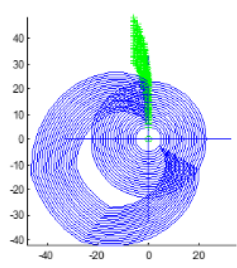

(a)

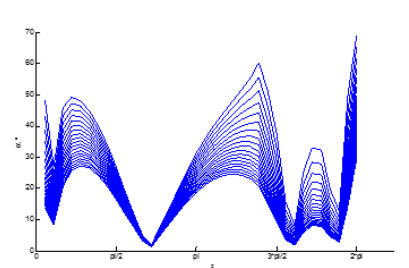

(b)

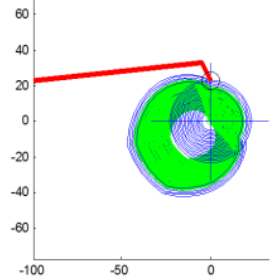

(c)

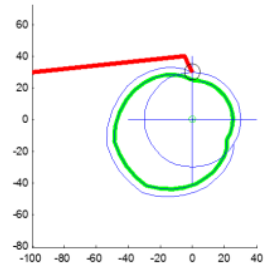

(d)

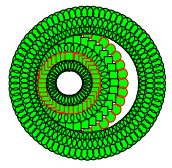

(e )

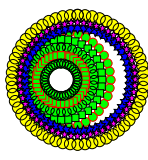

(f)

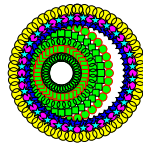

(g)

Figure 4. Cam profile automatically produced by the computer: a) the growth of cam profile; b) the pressure angle decreases until $\leq 30^{\circ}$ with the increase of $r 0$; $c$ ) the growth of cam profile with swing follower; d) final profile of the cam; e) cam by connecting points; f) cam with layers of different materials; g) cam with hybrid materials.

\subsection{Formation of structures}

The design processes of these examples in Section 2 can be analysed using many design theories. For example, the chamber consists of a group of parts, which can be understood as function decomposition and mapping sub-functions onto working structures. The design process of cam mechanisms and linkage mechanisms can be understood as behaviours-structure mapping of FBS framework. The innovative design of the rotary target and flexible linkage can be explained using TRIZ inventive principles and C-K theory. The flapping wing design can be taken as an analysis process of FBS and many other design theories. In this paper, these design examples are be analysed by analogy with four steps from DNA to proteins. 


\subsubsection{IC chamber}

In the chamber example, the selection of parts is made based on the working principle selected. When physical vapour deposition (PVD) is selected, the target is selected as source material to deposit and relative parts are predefined, such as target support, magnetron rod, substrate and the like. Once PVD is decided as the working principle, the chamber configuration is determined and the sketch of a PVD chamber is automatically produced by connecting existing structure patterns. These structures patterns are building blocks of chambers, including the source target, the target support, the substrate, the substrate support, the substrate transport assembly, the cylinder chamber, the magnetron assembly and several other parts. The connections of building blocks are predefined, so a sketch can be automatically produced in the case of routine design.

Connections include connecting pairs between the parts and elements, such as the contact pair and sealing pair between up-part of a cylinder and down-part of a cylinder, and all other contact pairs. The connections are predefined on the computer.

The design of the rotary target requires new structure patterns, including the gears to rotate the target support, the cross-bearing to enable the target support to rotate around the cylinder, the sealing members between the rotary support and the stationary cylinder to isolate the gas fluid inside the cylinder and the environment air outside the cylinder, the motor to drive the gears and the frame to fix the motor to the outer surface of the cylinder, all of which are outside the predefined building blocks on a computer. So this innovative design task is taken by human designers and the draft drawing of embodiment design is also produced by human designers.

Formation of structures refers to the embodiment of a chamber structure by drawing a detail sketch of each structure pattern with a particular form for a particular functionality. The embodiment design is produced by manually drawing on a computer because it is more complex and difficult to program codes to draw the chamber on computers than to manually draw the chamber on a computer.

Pattern-oriented strategy is used in chamber design because there is no strict mapping between behaviours and structures. It is the processing parameters that are the key parameters to determine the quality of the deposited films. Hence, the structural parameters are mainly decided based on experience. The building blocks are parts in these design examples. Design automation is possible only for routine design by predefining all structure details, which costs a lot of time and money.

The design process of the chamber can be described using similar steps in biology:

- Step 1: interpret information: a set of building blocks and computing is identified with a PVD chamber.

- $\quad$ Step 2: define a sequence of building blocks: the connections between relative building blocks are predefined on computers for routine design, while the connections for innovative design are defined by designers.

- $\quad$ Step 3: connect building blocks: the chamber configuration is produced.

- Step 4: formation: the embodiment of the chamber is produced by detail the shapes and forms as functional structures.

In this example, there is no transformation from the information to materials. Step 1 to step 2 is the transform from information to the selection of building blocks. Step 3 is to form the raw structure and step 4 is to form the fine structure. The building blocks are part structures in this example. The embodiment is manually drawn on the computer.

\subsubsection{Flapping wings}

Similar steps can be also used to describe the process of formation of the flapping wings. In the flapping wing example, the formation of structures is summarized as follows.

- Step 1: Information: structure patterns as structure information. The eight-bar linkage mechanism is selected. Gears are selected to transport motion.

- $\quad$ Step 2: Building blocks: including linkage mechanisms, gears, motors and the like.

- Step 3: Connection: contact pairs are made between gears and the linkages, between motors and the bars and the motor and the gears, and other contact pairs required to make a whole wing.

- Step 4: Formation: the embodiment of structures by detailing bars, gears and the body frame into particular forms to achieve the motion requirement and the strength requirement.

The building blocks are structure patterns including linkage mechanisms, gears, motors and the like. The embodiment of the flapping wings is manually drawn on a computer. 


\subsubsection{Cam and linkage mechanisms}

In these examples, the formation of structures can be also summarized as follows.

- $\quad$ Step 1: Information: displacement requirement, and selection of structure patterns, including cam mechanisms, a crank-rocker mechanism and a crank-slide mechanism.

- Step 2: Building blocks: points or tine masses.

- Step 3: Connection: points or masses are connected to each other.

- Step 4: Formation: the embodiment of structures by detailing the cam profile and the bars to meet the strength requirement and other constraints.

There is a strict mapping relation between behaviours and structures. All structural parameters are computed according to kinematic requirements. All cam and linkage examples are produced automatically by computers based on four rules and the same codes. The building blocks are points or masses that are connected to form structures. The whole process is automatic.

The processes of the formation of cam profiles and linkage profiles bare similarity with the process of gene transcription and translation: (1) to read the information of behaviours and to create a discrete point set $s_{\mathrm{i}}$, or a reduced discrete point set; (2) to transport the points to a new position by Rotation operations; (3) to find the joints' position by the Centre rule; (4) and finally to draw the sketch (the structure) by Connection rules. Therefore, the Rotation, Centre and Connection rules are translation process from reciprocating motion specification to mechanisms.

\subsection{Discussion}

The formation of structures follows similar steps in biology: read information, define the sequence of building blocks, connect the building blocks, and finally form structures by assembling building blocks. In biology, the formation of structures is an autonomous process in a complex biological, chemical and physical environment. Computers cannot simulate such an environment but can provide tools that simulate part of the formation process to assist designers with the design of structures. The process can suit for automation, partly automation or manual operation depending on the design strategy to use and the building blocks to assemble structures. Design tools based on structure patternoriented strategy suit for partly automation of routine design but not for innovative design, while design tools applying kinematic-governed computing and tine masses as building blocks suit for automation of the design of mechanisms consisting of bars, joints, cams and similar members. In many cases, structural parameters are decided based on experiments and simulations, in which case part of structures such as linkage mechanisms and cam mechanisms can be automatically produced.

It is very expensive to develop the design automation systems for each particular products, and it is impossible for innovative design unless building blocks are very small components and elements that can be used to construct any form using simple rules to meet particular design requirements.

\section{RELATED WORKS}

\subsection{Autonomous assembly of structures}

Cellar automata and genetic algorithms are often used to automatically generate particular structures such as tall buildings by using predefined structural patterns as building blocks (Kicinger and Arciszewski, 2007; D'Souza and Simpson, 2003). A cellular automaton is a simple computational mechanism that changes the state of each cell on a grid based on the state of adjacent (or nearby) cells according to transformation rules. In cellular automata, the main point is the decision rules to take the next action. The genetic algorithms currently are used to generate new structural parameters based on a population pool. The basic concept of GAs is to simulate processes in the natural system for evolution. Both cellular automata and genetic algorithms explain how behaviours mapping onto structures but not how structures are formed. In current research and engineering practice, human intervention is necessary to design complex mechanical devices. However, the idea of using simple discrete rules and the idea of using concepts in biology contribute to the use of biological concepts presented in this paper, although in an entirely different focus.

Many other methods have been used for mapping functions to structures. These methods include shape grammar (Jowers, Earl and Stiny, 2019; Stouffs, 2018), design grammar (Rudolph, 2005; Oster and McCormack, 2011), graph based representation and design rules (Munzer, Helms and Shea, 2013), spatial grammar (van Doepen and Shea, 2019), first-order logic and Boolean operation, Physics-Based 
Reasoning for grammar creation (Sen, Summers and Mocko, 2013) and similar methods. In these cases, grammar rules guide the formation of products by using predefined shapes as building blocks. Some design methods combine many mathematic algorithms and AI and IT technology to automate the design process. Among these methods, KBE is a key technology to automate the design process of complex mechanical systems. The principle of this kind of design systems is recording the design process on computers. For example, the knowledge and design procedure of aircraft, cars, small satellites and mould parts are recorded as software code so that repetitive design can be produced on computers (La Rocca and van Tooren, 2007; Colombo, Facoetti, Gabbiadini and Rizzi, 2010). The core of these methods is using software technology to build product templates including geometric description and the bill of material. The basic idea behind these methods is a predefined-template strategy. The good side of this strategy is a rapid response to the task requirements for specified products whose design process have been pre-recorded on computers. The shortness of this strategy is the lack of flexibility adapting to major structural modification and different design tasks.

Another way to approach autonomous assembly is to construct cubic robots as agent building blocks and modelling the assembly of a structure as position control of robot agents (Cabral, Givigi, and Jardine, 2020). This approach is to use agents to control the formation of structures.

\subsection{Programmable structures}

Recent emerging researches provide technologies to form structures from materials. For example, programmable things like programmable maters/materials/surfaces (Peraza Hernandez, 2016) and DNA origami (Praetorius et al, 2017) provide methods to make tailored materials to form predefined structures or to make a set of building blocks to form a chair and the like by self-assembly and selforganization under certain environments, such as the fluid flow, the temperature, the light and the force. In these cases, the structures are predefined through computing and programming.

\subsection{Self-organisation}

Research on self-organization is mainly a major concern of complex systems (CS). CS means that a system going into a complex stage becomes self-organized (Ashby, 1962). It studies how parts of a system and their relationships gives rise to collective behaviours of a system and how a system and its environment interrelate with each other (Bar-Yam, 2008). The operation of any part of a system affects the operation of other parts and vice versa. A typical example of complex systems is CyberPhysical Systems (CPSs). CPSs consist of a large number of computational components and physical components including biological components (Baheti, 2011). Operations of all components and subsystems are integrated into a single system and the system is monitored and/or controlled by a computational core with feedback loops for coordinating both physical processes and computational processes to adapt to emerging changes (Lee, 2008). Computing is integrated into every physical component and, in some cases, into materials to give systems the ability to behave in a much flexible and proper way. In these cases, the building blocks are sub-systems of an intelligent system, while mathematics, control and artificial intelligence (AI) are the main approaches for self-organization of a complex system.

\section{CONCLUSION AND DISCUSSION}

The gene transcription and translation process is a generic framework to describe both the formation of structures of products in engineering design and the formation of structures in biology. The process can be represented as four steps in general: information interpretation, selection of building blocks, the connection of building blocks, and formation of structures. The key step of the formation process is to assemble building blocks for both structures in engineering and in biology. Building blocks in biology are amino acids while they are structures in design. The autonomous degree of the formation process depends on the level of building blocks. The reuse degree of the building blocks depends on the level of building blocks too. In biology, structures of proteins are self-organized, so one way towards design automation is to use lower-level building blocks.

When parts and elements are taken as building blocks, the partial automation design process can be established for routine design based on the predefined description of existing structure patterns but not for creative design, as in the case of the chamber design example. The parts and elements can be reused only for products similar in shapes and working principles. 
When particular types of mechanisms are taken as building blocks, predefined methods are needed for the formation of different types of mechanisms, as in the case of a flapping wing. For example, the formation process of a six-bar linkage mechanism differs from an eight-bar linkage mechanism. Therefore, the formation process of a particular type of mechanisms can rarely be reused for different motion requirement. When the building blocks are points or tine masses, different types of mechanisms can be produced based on the same simple rules with an autonomous formation process and the reuse level of building blocks is high, as in the cases of the design of mechanisms.

The four steps of formation of structures are distributive both in space and in time and they can be reused repetitively to form a single structure at different levels. This means that distributive design tools rather than integrated design tools are effective assistants to designers, particularly for innovative design and creative design, something like toolboxes in Matlab or industrial robots for manufacturing automation and intelligent manufacturing. The further work is to identify and define a library of design building blocks and to develop distribute design tools to support partially autonomous design.

\section{ACKNOWLEDGMENTS}

This research is supported by Beijing Natural Science Foundation (Grant No. 3172014).

\section{REFERENCES}

Ashby, W.R. (1962), "Principles of the self-organizing system”, In: von Foerster, H. and Zopf, Jr. G.W. (Ed.), "Principles of Self-Organization: Transactions of the University of Illinois Symposium", Pergamon Press: pp. 255- 278.

Baheti, R., Gill, H. (2011), “The impact of control technology”, In: Samad, T. and Annaswamy, A.M. (Ed.), Available at: www.ieeecss.org.

Bar-Yam, Y. (2008)," General feature of complex systems”, http://www.eolss.net/sample-chapters/c15/E1-2901-00.pdf

Cabral, K.M., Givigi, S.N. and Jardine, P.T. (2020), "Autonomous assembly of structures using pinning control and formation algorithms", 2020 IEEE International Systems Conference (SysCon), Montreal, QC, Canada, 2020, pp. 1-7. https://dx.doi.org/10.1109/SysCon47679.2020.9275901

Colombo, G., Facoetti, G., Gabbiadini, S. and Rizzi, C. (2010), "Knowledge-based system for guided modelling of sockets for lower limb prostheses”, Computer Aided Design and Applications, Vol. 7, No. 5, pp. 723-737.

D'Souza, B., Simpson, T. W. (2003), “A genetic algorithm based method for product family design optimization”, Engineering Optimization, Vol. 35, No. 1: pp. 1-18.

Jowers, I., Earl, C. and Stiny, G. (2019), "Shapes, structures and shape grammar implementation", Computer Aided Design, Vol. 111, pp. 80-92. https://doi.org/10.1016/j.cad.2019.02.001

Kicinger, R., Arciszewski, T. (2007), "Breeding Better Buildings”, American Scientist, 95: pp. 502-508.

Kimmel, C.A., Buelke-Sam, J. (Ed.) (1994), Developmental Toxicology, Raven press, New York. http://homepages.rpi.edu/ bellos/transcription.htm.

La Rocca, G. and van Tooren, M.J.L. (2007), "Enabling distributed multi-disciplinary design of complex products: a knowledge based engineering approach", Journal of Design Research, Vol. 5, No. 3, pp. 333-352.

Lee, E.A. (2008), “Cyber Physical Systems: Design Challenges”, Technical Report No. UCB/EECS-2008-8, http://www.eecs.berkeley.edu/Pubs/TechRpts /2008 /EECS-2008-8.html, January 23.4.

Munzer, C., Helms, B. and Shea, K. (2013), "Representations into Boolean Satisfiability Problems for Computational Design Synthesis”, Journal of Mechanical Design, Vol. 135, 101001, pp. 1-13.

Peraza Hernandez, E. A., Hartl, D. J., Malak, R. J., Jr., Akleman, E., Gonen, O., and Kung, H. (2016). "Design Tools for Patterned Self-Folding Reconfigurable Structures Based on Programmable Active Laminates." ASME. J. Mechanisms Robotics, 8(3): 031015, pp. 1-12. https://doi.org/10.1115/1.4031955

Praetorius, F., Kick B., Behler, K.L., Honemann, M.N., Weuster-Botz, D. and Dietz, H. (2017) "Biotechnological mass production of DNA origami", Nature, Vol. 552, No. 7, pp. 84-87.

Rudolph, S. (2005), "Space station design rules", Aerospace engineering, pp. 43-45.

van Diepon, M. and Shea, K. (2019), "A Spatial Grammar Method for the Computational Design Synthesis of Virtual Soft Locomotion Robots", Journal of Mechanical Design, Vol. 141, 101402, pp. 1-10.

Stouffs, R. (2018), "Implementation issues of parallel shape grammars", Artificial Intelligence for Engineering Design, Analysis and Manufacturing, Vol. 32, No. 2, pp. 162-176. 